\title{
LOC101930370/MiR-1471 Axis Modulates the Hedgehog Signaling Pathway in Breast Cancer
}

\author{
Xin Liu ${ }^{\mathrm{a}}$ Tong Zhao ${ }^{\mathrm{a}} \quad$ Xue Bai ${ }^{\mathrm{a}}$ Mingqi Lia Jingli Ren ${ }^{\mathrm{b}}$ Mengxue Wang ${ }^{\mathrm{a}}$ \\ Ranchen $\mathrm{Xu}^{\mathrm{a}}$ Shuqian Zhang ${ }^{\mathrm{a}}$ Huimin $\mathrm{Li}^{\mathrm{a}}$ Yingying Hu $\mathrm{Hu}^{\mathrm{c}}$ Liangjun $\mathrm{Xie}^{\mathrm{a}}$ \\ Ying Zhang $^{\text {a }}$ Lida Yang $^{\mathrm{a}}$ Chaoqi Yan ${ }^{\mathrm{d}}$ Yong Zhang ${ }^{\mathrm{a}, \mathrm{e}}$
}

aDepartment of Pharmacology (the State-Province Key Laboratories of Biomedicine-Pharmaceutics of China, Key Laboratory of Cardiovascular Research, Ministry of Education), College of Pharmacy, Harbin Medical University, Harbin, b Department of pharmacology, Luohe Medical College, Luohe, 'Department of Pharmacy, The First Affiliated Hospital of Harbin Medical University, Harbin, dDepartment of Breast Surgery, The Second Affiliated Hospital of Harbin Medical University, Harbin, eInstitute of Metabolic Disease, Heilongjiang Academy of Medical Science, Harbin, China

\section{Key Words}

MiR-1471 • LOC101930370 • SHH • Gli-1 • Hedgehog pathway • Breast cancer

\begin{abstract}
Background/Aims: Non-coding RNAs (ncRNAs) play vital regulatory roles in many tumors. However, the functional roles of these transcripts responsible for their dysregulation in breast cancer $(B C)$ are not thoroughly understood. Methods: We examined the expression of microRNA miR-1471 in BC specimens. Online analysis tools predicted that IncRNA LOC101930370 might act as an endogenous 'sponge' by competing for miR-1471 binding targets. Luciferase assays were used to prove the interaction of LOC101930370, miR-1471 and $\mathrm{SHH}$. Edu, wound-healing and transwell assays were used to verify the contribution of miR-1471 and LOC101930370 on MCF-7 cells proliferation and metastasis. Gain and loss of function studies were performed to evaluate the relevance of Hedgehog pathway with LOC101930370/miR-1471 regulating axis in MCF-7 cells. Results: The expression of miR-1471 was markedly downregulated in BC. Inhibition of miR-1471 by LOC101930370 was proved by luciferase assay. Knockdown of LOC101930370 suppressed BC cells progression. MiR-1471 inhibitor resulted in a more aggressive metastasis of MCF-7 cells. Moreover, SHH and Gli-1 expression were significantly suppressed by LOC101930370 knockdown, and upregulated by miR-1471 inhibitor transfection. Conclusions: Collectively, our study reveals the interaction between LOC101930370 and miR-1471 for the first time. LOC101930370 positively regulates the expression of SHH by sponging miR-1471, which sheds new light on IncRNA-directed diagnostics and therapeutics in BC.

$X$. Liu and T. Zhao contributed equally to this work.




\section{Cellular Physiology Cell Physiol Biochem 2018;48:1139-1150 \begin{tabular}{l|l} 
and Biochemistry Published $10.1159 / 000491980$ & $\begin{array}{l}\text { C } 2018 \text { The Author(s). Published by S. Karger AG, Basel } \\
\text { www.karger.com/cpb }\end{array}$
\end{tabular}}

Liu et al.: LOC101930370/miR-1471 Axis Regulates Breast Cancer Metastasis

\section{Introduction}

Breast cancer (BC) is a malignant neoplasm originating from breast tissue and the most common cause of death of women throughout the world [1, 2]. A central deficiency in our knowledge of cancer concerns how genomic changes drive the proteome to execute phenotypic characteristics. Advances in functional genomics have revealed that the human genome is actively transcribed. However, vast majority of the transcripts are non-coding RNA (ncRNA) including microRNAs and long non-coding RNAs (lncRNAs).

MicroRNAs (miRNAs), which are $~ 22$ nucleotides in length, play important roles in the regulation of target gene expression by base pairing to complementary sites in 3 '-untranslated regions (3'UTR) of the target mRNA [3, 4]. The microRNAs may act as oncogenes, tumor suppressors and modulators of tumor proliferation, invasion, apoptosis and therapy resistance [5-7]. In the case of BC, cDNA microarrays using probes for miRNAs have revealed that sets of miRNAs are differentially expressed in primary invasive carcinoma. Among them, some data have indicated that miR-1471 is related to BC oncogenesis [8]. However, the molecular mechanisms utilized by miR-1471 in BC are totally unclear. To clarify the potential role of miR-1471 in BC, we used TargetScan and microRNA.org to predict the putative targets of miR-1471. One of the most conserved is Sonic hedgehog (SHH), a crucial protein involved in Hedgehog pathway. Previous studies have shown that activation of the Hedgehog pathway can promote myofibroblast differentiation, stromal hyperplasia, and extracellular matrix (ECM) production, which facilitate the EMT process, invasion, and metastasis of BC [9-11]. Aberrant expression of $\mathrm{SHH}$ is implicated in the development of several tumors, including brain, pancreas, and BC[12-14]. Glioma-Associated Oncogene Family Zinc Finger 1 (Gli-1) is a transcriptional factor of the Hedgehog pathway [15-17]. Recent study has shown that the expression of Gli- 1 is higher in TNBC cell lines and promotes angiogenesis by transcriptional modulation of the VEGFR2 [18]. Gli-1 levels also associate with the poor prognosis of BC [19-21]. However, whether miR-1471 regulates Hedgehog pathway by targeting SHH has not been clarified.

Unlike microRNAs, lncRNAs are larger than 200 bps in length. They have emerged as key players in a wide variety of cellular processes, including carcinogenesis and tumour growth $[22,23]$. One of well-characterized mechanisms is the lncRNA-mediated gene regulation through complementary interaction with miRNAs [24]. For instance, GAS5 inhibits the expression of miR-21 through the competing endogenous RNA mechanism [25, 26]. LncRNA MIR31HG exhibits oncogenic property in pancreatic ductal adenocarcinoma and is negatively regulated by miR-193b [25]. However, whether IncRNAs are involved in the down-regulation of miR-1471 in BC remains unclear.

Taken together, in the present study, we investigated the expression and roles of IncRNA, microRNA-1471, SHH and transcription factor Gli-1 in BC. Meanwhile, the interaction among lncRNA, microRNA-1471, SHH and Gli-1 in regulation of BC metastasis and the possible mechanisms were also revealed.

\section{Materials and Methods}

\section{Patients and specimens}

Human Breast cancer specimens (breast tumor and adjacent non-tumor tissue) were obtained from patients diagnosed with mammary cancer who underwent surgery at the Second Affiliated Hospital of Harbin Medical University (Harbin, China), from February 2017 to June 2017. The specimens from patients were frozen in liquid nitrogen immediately after the cancer ectomy, then stored at $-80^{\circ} \mathrm{C}$. A total of 15 patients were enrolled and the clinicopathological information of patients is shown in Table 1. The research methods in our study were approved by the Ethics and Scientific Committees of Harbin Medical University. Written informed consents were obtained from all the enrolled patients (HMUIRB20170017). 


\section{Cellular Physiology Cell Physiol Biochem 2018;48:1139-1150 \begin{tabular}{l|l} 
and Biochemistry Published 10.1159/000491980 & $\begin{array}{l}\text { (c) } 2018 \text { The Author(s). Published by S. Karger AG, Basel } \\
\text { www.karger.com/cpb }\end{array}$ \\
\hline
\end{tabular}}

Liu et al.: LOC101930370/miR-1471 Axis Regulates Breast Cancer Metastasis

Cell culture

Four breast cancer cell lines (MCF-7, MDA-MB-231, BT474, SKBR3) were used (Shanghai fuxiang biotechnology Co., Ltd). MCF-7, MDA-MB-231 and SKBR3 were grown in Dulbecco's modified eagle's medium (DMEM) containing $10 \%$ fetal bovine serum (FBS) at the temperature of $37{ }^{\circ} \mathrm{C}$ in $5 \% \mathrm{CO}_{2}$. BT-474 was cultured in Roswell Park Memorial Institute 1640 (RPMI-1640) medium containing 10\% fetal bovine serum (FBS) at the same incubator. For transfection, The MCF-7 cells were starved in serum-free medium for 24 $\mathrm{h}$, and then transiently transfected with $100 \mathrm{nM}$ miR-1471 mimics, miR-1471 inhibitors or negative controls (RiboBio Co., Ltd., Guangzhou, Guangdong, China), and si-lncRNA (LOC101930370 siRNA) or si-NC (non-sense control of silncRNA) (Invitrogen, USA). X-treme GENE siRNA transfection reagent (Shanghai GenePharma., Ltd, China) was used according to the manufacturer's instructions.

\section{Quantitative real-time PCR}

QRT-PCR were used to determine the expression levels of LOC101930370, miR-1471, miR-718, SHH and Gli1 transcripts. Total RNA was extracted by TRIzol reagent (Invitrogen, Carlsbad, USA) according to manufacturer's instructions. The SYBR Green Real-time PCR Master Mix Kit (TOYOBO) was used in real-time PCR for relative quantification of RNAs. GAPDH and U6 were used as an internal control.

The following primers were used:

LOC101930370: Forward, 5' -ACCTCTCCCTTGTGGAGGAT-3'; Reverse, 5' -CAGGTCCCCTCTCTTGTCAG-3'. miR-1471: RT, 5'-GTCGTATCCAGTGCGTGTCGTGGAGTCGGCAATTGC

ACTGGATACGACACACCT-3'; Forward, 5' $\quad$-TGGACCCTGGTCTACTCCTG-3'; $\quad$ Reverse, $\quad 5^{\prime}$ -CAAGAGCCCCTGTACAGCAT-3'.

miR-718: RT, 5' - GTCGTATCCAGTGCGTGTCGTGGAGTCGGCAATTGCA

CTGGATACGACCGACGC-3'; Forward, 5' - GTTATTCTTCCGCCCCGCCAG-3'; Reverse, 5'GGCTCGTGGAGTCGGCAATTG-3'.

SHH: Forward, 5' -CCAATTACAACCCCGACATC-3'; Reverse, 5'-CTCTGAGTGGTGGCCATCTT-3'.

Gli-1: Forward, 5'-GTGCAAGTCAAGCCAGAACA-3'; Reverse, 5' - GGGGCCTGACTGGAGATATT-3'.

GAPDH: Forward, 5' -AAGAAGGTGGTGAAGCAGGC-3'; Reverse, $5^{\prime}$-TCCACCACCCAGTTGCTGTA-3'.

U6: Forward, 5'-GCTTCGGCAGCACATATACTAAAAT-3'; Reverse, 5' -CGCTTCACGAATTTGCGTGTCAT-3'

\section{Western blotting}

MCF-7 cell protein samples were grinded in 200 ul of ripa containing a cocktail of protease inhibitor. Lysates were centrifuged at $13500 \mathrm{r} / \mathrm{s}$ for $20 \mathrm{~min}$. Protein concentration in the supernatant was measured by BCA Protein Assay (Beyotime, Shanghai, China). Equal amounts of protein samples (80 ug) were fractionated on a $10 \%$ SDS-PAGE gel electrophoresis and transferred onto nitrocellulose membranes. Then the membranes were blocked in the defatted milk (5\%) for $2 \mathrm{~h}$ and incubated on the shaker at $4{ }^{\circ} \mathrm{C}$ overnight with the primary antibodies of SHH (Abcam, Cambridge, MA, USA), Gli-1 (Abcam, Cambridge, MA, USA), vimentin (Wanlei, Inc., Shenyang, China), E-cadherin (Wanlei, Inc., Shenyang, China), $\alpha$-SMA (Wanlei, Inc., Shenyang, China) with GAPDH (Zhongshanjinqiao, Inc., Beijing, China) as an internal control. Appropriate secondary antibody was (Invitrogen) incubated for $1 \mathrm{~h}$ at room temperature. Protein bands were visualized and quantified by Odyssey Infrared Imaging System (LI-COR, Lincoln, NE, USA).

\section{Cell proliferation}

Cell proliferation was measured using a Cell Counting Kit-8, CCK8 (Dojindo, Kumamoto, Japan). MCF-7 cells were plated in 24-well plates at $3 \times 10^{5}$ cells/well. Then cells were incubated in $10 \%$ CCK8 which was 


\section{Cellular Physiology Cell Physiol Biochem 2018;48:1139-1150 \begin{tabular}{l|l} 
DOI: 10.1159/000491980 & $\begin{array}{l}\text { O } 2018 \text { The Author(s). Published by S. Karger AG, Basel } \\
\text { www.karger.com/cpb }\end{array}$
\end{tabular}}

Liu et al.: LOC101930370/miR-1471 Axis Regulates Breast Cancer Metastasis

diluted in normal culture medium at $37^{\circ} \mathrm{C}$ for color conversion. Proliferation rates were determined at $24 \mathrm{~h}$ after transfection.

\section{EdU assay kit}

Cells plated on coverslips in 6-well culture plates were treated as experiment design. The proliferation of MCF-7 cells were detected by EdU kit (RiboBio, China) according to the manufacturer's instructions. A fluorescence microscope (Olympus, Japan) was used to acquire the images. Nuclei that double labeled with EdU and DAPI were considered to be positive cells.

\section{Cell migration and Invasion}

To analyze wound healing, the cells were seeded in six-well plates with complete DMEM medium. After $48 \mathrm{~h}$, the cell monolayer was wounded using a plastic pipette tip. Then the cells were rinsed with PBS and cultured with serum-free DMEM for $24 \mathrm{~h}$. The wound closure was observed and photographed under a microscope. For the Transwell assays, 8- $\mu$ m pore size chambers (Corning, NY, USA) were used with an insert coated with Matrigel (BD Bioscience). Twenty-four hours after transfection, $1 \times 10^{5}$ cells in serumfree medium were added to the upper chamber. The lower chamber was filled with 10\% FBS DMEM. After $24 \mathrm{~h}$ of incubation, the cells remaining on the upper surface of the membrane were removed, whereas the cells that had invaded through the membrane were fixed with $100 \%$ methanol for 15 minutes, stained with $0.1 \%$ crystal violet for 20 minutes. Images of MCF-7 cells were taken under a phase-contrast microscope (Olympus, Japan).

\section{Immunofluorescence staining and immunocytochemistry}

For immunofluorescence staining, MCF-7 were fixed with $4 \%$ buffered paraformaldehyde in PBS. Blocking solution (1\% BSA and $0.1 \%$ Triton-X in PBS) was used to penetrate and incubate fixed cells at room temperature for $2 \mathrm{~h}$. Primary antibodies against SHH (1:200) and Gli-1(1:200) were placed in PBS overnight at $4{ }^{\circ} \mathrm{C}$, followed by incubation with the appropriate secondary antibody (Invitrogen) for $1 \mathrm{~h}$ at room temperature. Then nuclei were stained using 4',6-diamidino-2-phenylindole (DAPI; Beyotime, Shanghai, China) for 20 mins at room temperature. Immunofluorescence was examined and analyzed by laser scanning confocal microscopy (FV300, Olympus, Japan).

\section{Statistical analysis}

Group data was described as the mean \pm S.E.M. Student's t-test or one-way ANOVA accompanied by Bonferroni's Multiple Comparison Test was used to analyze comparisons. $p<0.05$ was considered to indicate a significant difference. Data were analyzed using the GraphPad Prism 5.0 software.

\section{Results}

\section{MiR-1471 is down-regulated in breast cancer}

RNA regulatory mechanisms have been connected to oncogenesis. Studies from whole genome and transcriptome analysis have indicated that miR-1471, miR-718 were downregulated in breast cancer [8]. To further validate the possible roles of these two miRNAs in BC, we evaluated the miR-1471 and miR-718 levels in 15 paired breast cancer and adjacent breast tissue samples using qRT-PCR. The results showed that miR-1471 was significantly ( 0.43 fold changes) decreased in tumor tissues compared to adjacent breast tissues (Fig. 1a). However, downregulation of miR-718 in BC tissues was not such apparent (Fig. 1b). We then tested the expression level of miR-1471 in several breast cancer cell lines (MCF-7, BT474, SKBR3, MDA-MB-231). Compared with non-tumor breast tissues, MCF-7 cells exhibited lowest expressed miR-1471 (Fig. 1c). Collectively, these results suggested that miR-1471 was down-regulated in $\mathrm{BC}$ cells and tissues. That might indicate a potential tumor suppressor role of miR-1471 in BC tumorigenesis.

LOC101930370 is a competing endogenous RNA (ceRNA) of miR-1471

Based on our recent studies [25], we selected lncRNAs connected to the control of miR-1471. Two databases LncTar and NCBI BLAST predicted that IncRNA LOC101930370 
Fig. 1. MiR-1471 was dysregulated in breast cancer. a. Relative miR-1471 expression levels in 15 paired breast cancer and adjacent breast tissue specimens determined by qRT-PCR. b. Relative miR-718 expression levels in 15 paired breast cancer and adjacent breast tissue specimens determined by qRT-PCR. c. MiR-1471 expression levels in four breast cancer cell lines, MCF-7, MDAMB-231, BT-474, SKBR3 and non-tumor breast

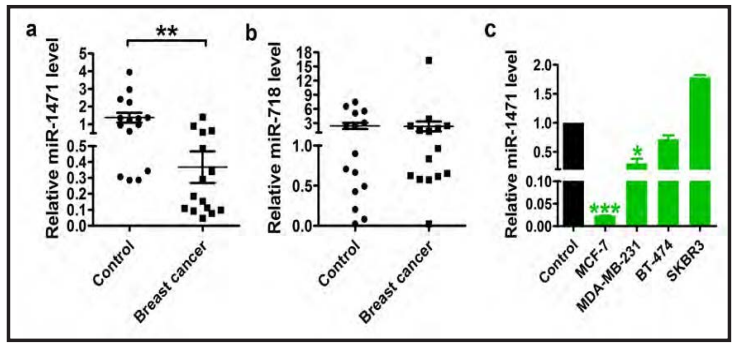
tissue, determined by qRT-PCR. ${ }^{*} \mathrm{p}<0.05^{* *} \mathrm{p}<0.01{ }^{* * *} \mathrm{p}<0.001$ vs. Control. $\mathrm{n}=3$ batches of cells. The data are presented as mean \pm SEM.

Fig. 2. LOC 101930370 is a ceRNA of miR-1471. A. Sequence complementarity between LOC101930370 and miR-1471. b. Luciferase reporter constructs containing the binding sites of LOC101930370 and miR-1471, or the mutated binding sites. c,d. Luciferase reporter assay showed that transfected miR-1471 could reduce the luciferase activity in wild-type (wt) of Luciferase constructs but not in mutant (mut) one. ${ }^{* *} \mathrm{p}<0.01, \# \# \mathrm{p}<0.01$ vs. miR-1471. e. LOC101930370

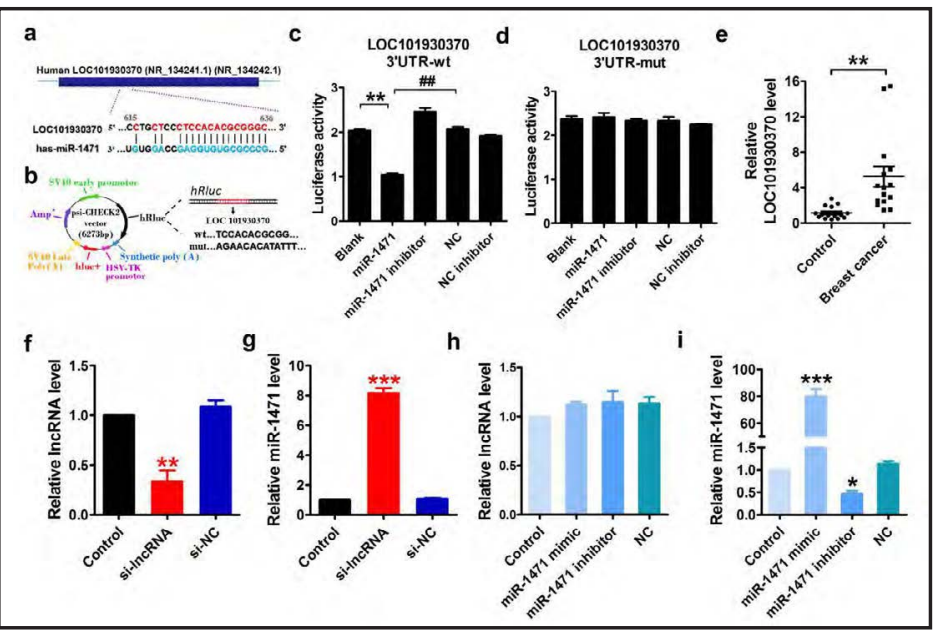
expression levels in 15 paired breast cancer and adjacent breast tissue specimens determined by qRT-PCR. f. LOC101930370 expression levels determined by qRT-PCR. g. MiR-1471 expression levels determined by qRT-PCR. h. The influence of miR-1471 mimic and inhibitor on expression of LOC101930370. i. MiR-1471 expression levels after miR1471 mimic and inhibitor transfection in MCF-7 cells. ${ }^{*} \mathrm{p}<0.05^{* *} \mathrm{p}<0.01{ }^{* * *} \mathrm{p}<0.001$ vs. Control. $\mathrm{n}=3$ batches of cells. The data are presented as mean \pm SEM.

was complementary binding with miR-1471 (Fig. 2a). Luciferase assay was used to identify the inhibitory effect of LOC101930370 on miR-1471 (Fig. 2b). The results demonstrated that miR-1471 dramatically inhibited the luciferase activity of the constructed plasmid containing wild-type (WT) LOC101930370, but not that of the mutant (mut) plasmid (Fig. $2 \mathrm{c}$ and d). We then tested the expression of LOC101930370 in tumor specimens from BC patients. The results suggested a significant upregulation of LOC101930370 in BC tissues (Fig. 2e). In addition, LOC101930370 expression was remarkably upregulated in MCF-7 cells (for all online suppl. material, see www.karger.com/doi/ 10.1159/000491980, Suppl. Fig. 1). Therefore, we proceeded to explore a potential regulatory axis involving LOC101930370 and miR-1471. To verify the regulated interaction of the two ncRNAs, we transfected MCF-7 with small interfering RNA against LOC101930370 (si-lncRNA). LOC101930370 level was significantly suppressed (Fig. 2f). Cellular miR-1471 levels were significantly increased after the si-lncRNA transfection (Fig. 2g). To determine if miR-1471 has feedback regulatory effects on LOC101930370, miR-1471 mimics, miR-1471 inhibitor, and nonsense control were transfected to MCF-7 cells. Significant overexpression or knockdown of miR-1471 did not affect cellular LOC101930370 levels (Fig. 2h and i). Collectively, LOC101930370 is associated with the reduction of miR-1471 in BC. 


\section{Cellular Physiology and Biochemistry}

Fig. 3. Knockdown of LOC101930370 inhibits MCF-7 cell proliferation. a. Representative images of Edu staining showing proliferation cells (stained in pink). Nuclei that double labeled with EdU (red) and DAPI (blue) were considered to be proliferative cells. Scale bar indicates $100 \mu \mathrm{m}$. b. The Edu positive cells were counted. c. MCF-7 cells viability were detected by CCK8 assay. d,f. The effect of LOC101930370 downregulation on the invasion of MCF-7 cells was assessed using transwell assay. Scale bar indicates $100 \mu \mathrm{m}$. e,g.

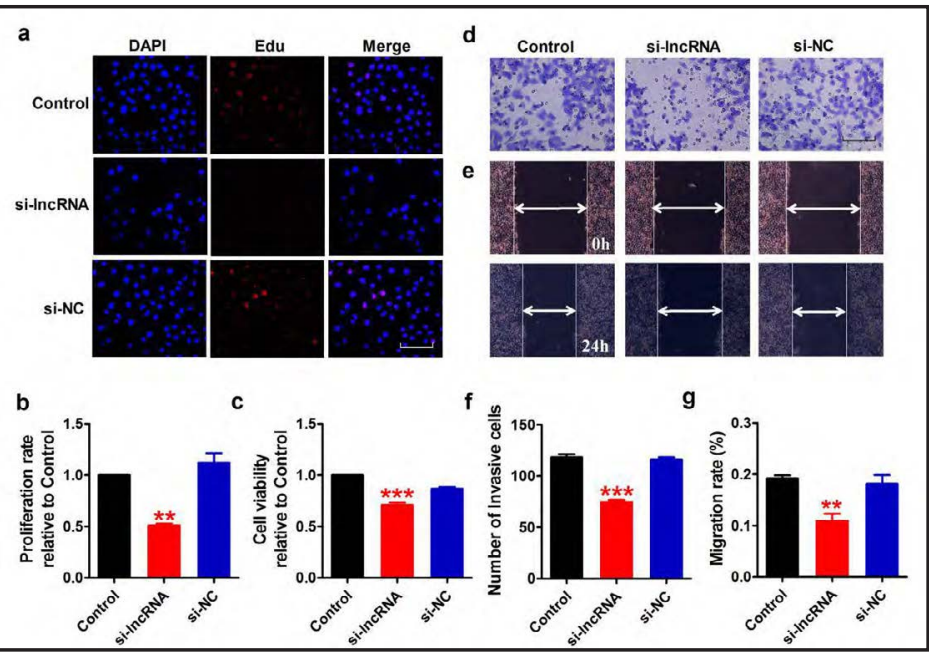
Wound healing assay showed that LOC101930370 siRNA resulted in a slower closing of scratch wounds. ${ }^{* *} \mathrm{p}<0.01^{* * *} \mathrm{p}<0.001$ vs. Control. $\mathrm{n}=3$ batches of cells. Data are expressed as mean \pm SEM.

\section{Knockdown of LOC101930370 inhibits MCF-7 cell proliferation and metastasis}

Next, we asked if decreasing LOC101930370 protected MCF-7 cells from malignancy. We transfected MCF-7 cells with siRNA against LOC101930370. 5-Ethynyl-2' -deoxyuridine (Edu) assay was performed for determining cell proliferation. The results showed that Edu positive cells were drastically reduced following LOC101930370 downregulation (Fig. 3a and b). Consistent with this, cell viability detected by CCK8 showed that LOC101930370 knockdown significantly decreased MCF-7 cell viability (Fig. 3c). To further determine whether LOC101930370 was associated with the progression of MCF-7, we analyzed the effect of LOC101930370 knockdown on the invasive behavior of MCF-7 cells. Using a transwell system, we found that the invasive ability of MCF-7 cells was significantly decreased following si-lncRNA mediated downregulation of LOC101930370 (Fig. 3d and f). In addition, the wound-healing assay showed that cells transfected with si-lncRNA underwent a slower closing of scratch wounds compared with the control groups (Fig. 3e and g). Taken together, these results suggeted that LOC101930370 siRNA might act as a tumor suppressing tool through inhibiting cell proliferation, migration and invasion in BC.

\section{MiR-1471 inhibitor enhances tumor growth and tumorigenesis}

Next, we sought to determine the effect of miR-1471 on the proliferation and tumorpropagating potential of MCF-7 cells. We conducted gain-of-function experiment using miR1471 mimics and loss-of-function experiments using miR-1471 inhibitor (Fig. 2i). After transfection, MCF-7 cells proliferation and viability were examined by Edu assay (Fig. 4a and b) and CCK8 (Fig. 4c). Transfected with miR-1471 mimic exhibited a significant decrease of cell proliferation and cell viability. But miR-1471 inhibitor caused a marked increase in the proliferation and viability of MCF-7 cells. Following the same treatment regime, we also examined cell invasion and migration. Invasion of MCF-7 cells was significantly reduced following miR-1471 mimic transfection as shown by transwell assay. While MCF-7 cell invasion increased after miR-1471 inhibitor transfection compared with control group (Fig. $4 \mathrm{~d}$ and $\mathrm{f}$ ). Also, the cell migration was suppressed by miR-1471 mimic, upregulated by miR1471 inhibition (Fig. 4e and g). Taken together, these results indicated the tumor suppressing roles of miR-1471 in MCF-7 cells, which was opposite to its ceRNA LOC101930370.

\section{SHH is the downstream target of miR-1471}

The next question is how LOC101930370/miR-1471 modulates the MCF-7 cell phenotypes. In an effort to identify the putative target of miR-1471, we used TargetScan 
Fig. 4. MiR-1471 inhibitor enhances tumor progression. a. Representative images of Edu staining showed proliferation cells (stained in pink). Nuclei that double labeled with EdU (red) and DAPI (blue) were considered to be proliferative cells. Scale bar indicates $100 \mu \mathrm{m}$. b. The Edu positive cells were counted. c. MCF-7 cells viability were detected by CCK8 assay. d,f. The effect of miR-1471 mimic and miR1471 inhibitor transfection on the invasion of MCF-7 cells was assessed using transwell assay. Scale bar indicates $100 \mu \mathrm{m}$. e,g. Wound healing assay showed that

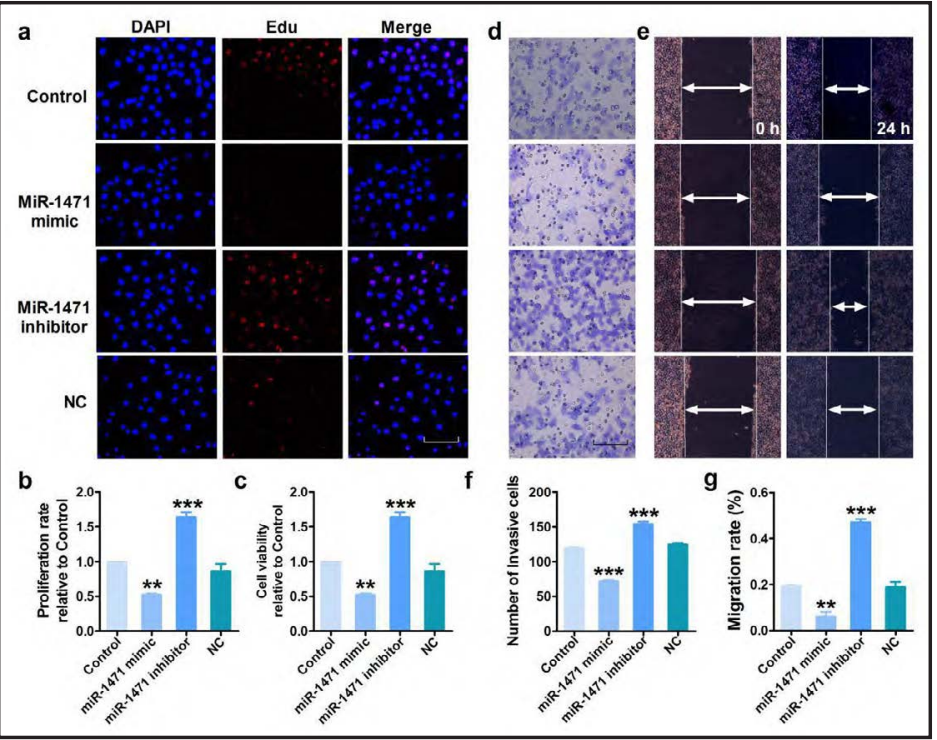
miR-1471 overexpression resulted in a slower closing of scratch wounds, miR-1471 inhibition caused a faster closing of scratch wounds. ${ }^{* *} \mathrm{p}<0.01{ }^{* * *} \mathrm{p}<0.001$ vs. Control. $\mathrm{n}=3$ batches of cells. Data are expressed as mean \pm SEM.

Fig. 5. SHH acts as a downstream target of miR-1471. a. Sequence complementarity between miR-1471 and SHH 3'UTR in human. b. Luciferase reporter constructs containing 3'UTR of SHH or mutated 3'UTR of SHH. c,d. Luciferase reporter assay showed that transfected miR1471 could reduce the luciferase activity in wild-type (wt) of SHH but not in mutant (mut) 3'UTR of SHH. ${ }^{* *} \mathrm{p}<0.01, \# \# \mathrm{p}<0.01$ vs. miR-

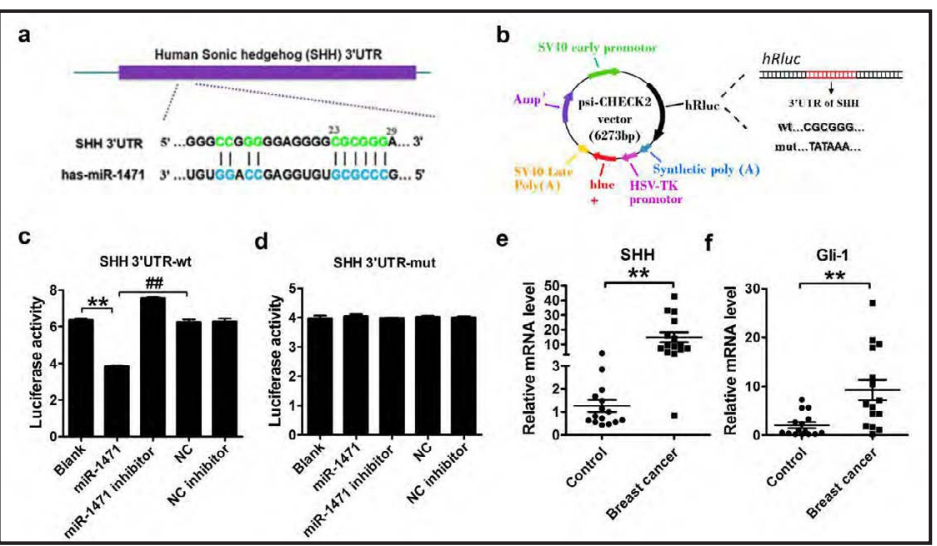
1471. e. SHH expression levels in 15 paired breast cancer and adjacent breast tissue specimens determined by qRT-PCR. f. Gli-1 expression levels in 15 paired breast cancer and adjacent breast tissue specimens determined by qRT-PCR. ${ }^{* *} \mathrm{p}<0.01 \mathrm{vs.}$ Control. $n=3$ batches of cells. The data are presented as mean \pm SEM.

and microRNA.org to predict its complementary binding targets. Our attention was focused on $\mathrm{SHH}$, a major pleiotropic developmental gene involved in Hedgehog signaling pathway (Fig. 5a). Together with its downstream transcription factor Gli-1, they control epithelialmesenchymal transition, proliferation, migration and invasion of different cancer cells [27]. Furthermore, we used luciferase assay to confirm the inhibitory effects of miR-1471 on SHH (Fig. 5b). As the results demonstrated, the luciferase activity of the constructed plasmid containing wild-type (WT) 3'UTR of SHH was inhibited by miR-1471, but not that of the mutant (Mut) 3' UTR (Fig. 5c and d). Our results showed that SHH and Gli-1 mRNA levels were significantly up-regulated in tumor specimens of BC patients (Fig. $5 e$ and $f$ ).

LOC101930370/miR-1471-mediates BC progression by regulation of SHH/Gli-1

Accordingly, we hypothesized that SHH may serve as a biologically relevant target of miR-1471 and participate in LOC101930370/miR-1471-mediated regulation of breast cancer development. As the downstream regulatory targets, si-lncRNA suppressed SHH and 
Gli-1 mRNA and protein expression in MCF-7 cellular nucleus, exhibited by qRT-PCR (Fig. 6a and b), WB (Fig. 6c and d) and immunofluorescence (Fig. 5e and f) (see online suppl. material, Suppl. Fig. 2) results. We also detected the levels of soluble SHH in the cell culture supernatants. The results showed that the secreted SHH was decreased by LOC101930370 siRNA transfection (see online suppl. material, Suppl. Fig. 3a). All these results suggested an important role of LOC101930370 in modulating SHH by competitively binding miR-1471.

\section{MiR-1471 inhibits Hedgehog signaling pathway by targeting SHH}

We then evaluated the influence of miR-1471 on SHH and Gli-1 expression using the similar transfection procedure. For this purpose, we transduced the MCF-7 cells with miR1471 mimic for overexpression and miR-1471 inhibitor for inhibition. Consistent with our prediction, SHH and Gli-1 transcripts were reduced after transfection of miR-1471 mimic, and increased by miR-1471 inhibitor (Fig. 7a and b). In agreement, western blot (Fig. 7c and d) and immunofluorescence (Fig. 7e and f) (see online suppl. material, Suppl. Fig. 4) analysis showed a decrease in SHH and Gli-1 protein levels with transfection of miR-1471 mimic over control group. A remarkable increase of these two protein expression was also observed after miR-1471 inhibitor transfection. Meanwhile, soluble SHH in the cell culture supernatants had a marked decreasing after miR-1471 mimic transfection, while increased by miR-1471 inhibitor (see online suppl. material, Suppl. Fig. 3b). Epithelial-mesenchymal

Fig. 6. Downregulation of LOC101930370 inhibits SHH and Gli-1 expression. QRT-PCR analysis of SHH (a) and Gli-1 (b) mRNA levels after LOC101930370 siRNA transfection. Western blot analysis of SHH (c) and Gli-1 (d) protein levels. Representative images of immunofluorescence staining for SHH (green) (e), Gli-1 (green) (f) in MCF-7 cells. The nuclei were stained in blue with DAPI. Scale bar indicates $100 \mu \mathrm{m}$. ${ }^{* * *} \mathrm{p}<0.001$ vs. Control. $n=3-5$ batches of cells. Data are expressed as mean \pm SEM.

Fig. 7. $\mathrm{SHH}$ and Gli-1 are the downstream targets of LOC101930370/ miR-1471 axis. QRT-PCR analysis of SHH (a) and Gli-1 (b) mRNA levels after miR-1471 mimic and inhibitor transfection. Western blot analysis of SHH (c) and Gli-1 (d) protein levels. Representative images of immunofluorescence staining for SHH (green) (e), Gli-1 (green) (f) in MCF-7 cells. The nuclei were stained in blue with DAPI. Scale bar indicates $100 \mu \mathrm{m} .{ }^{*} \mathrm{p}<0.05$ $* * * \mathrm{p}<0.001$ vs. Control. $\mathrm{n}=3-5$ batches of cells. Data are expressed as mean \pm SEM.
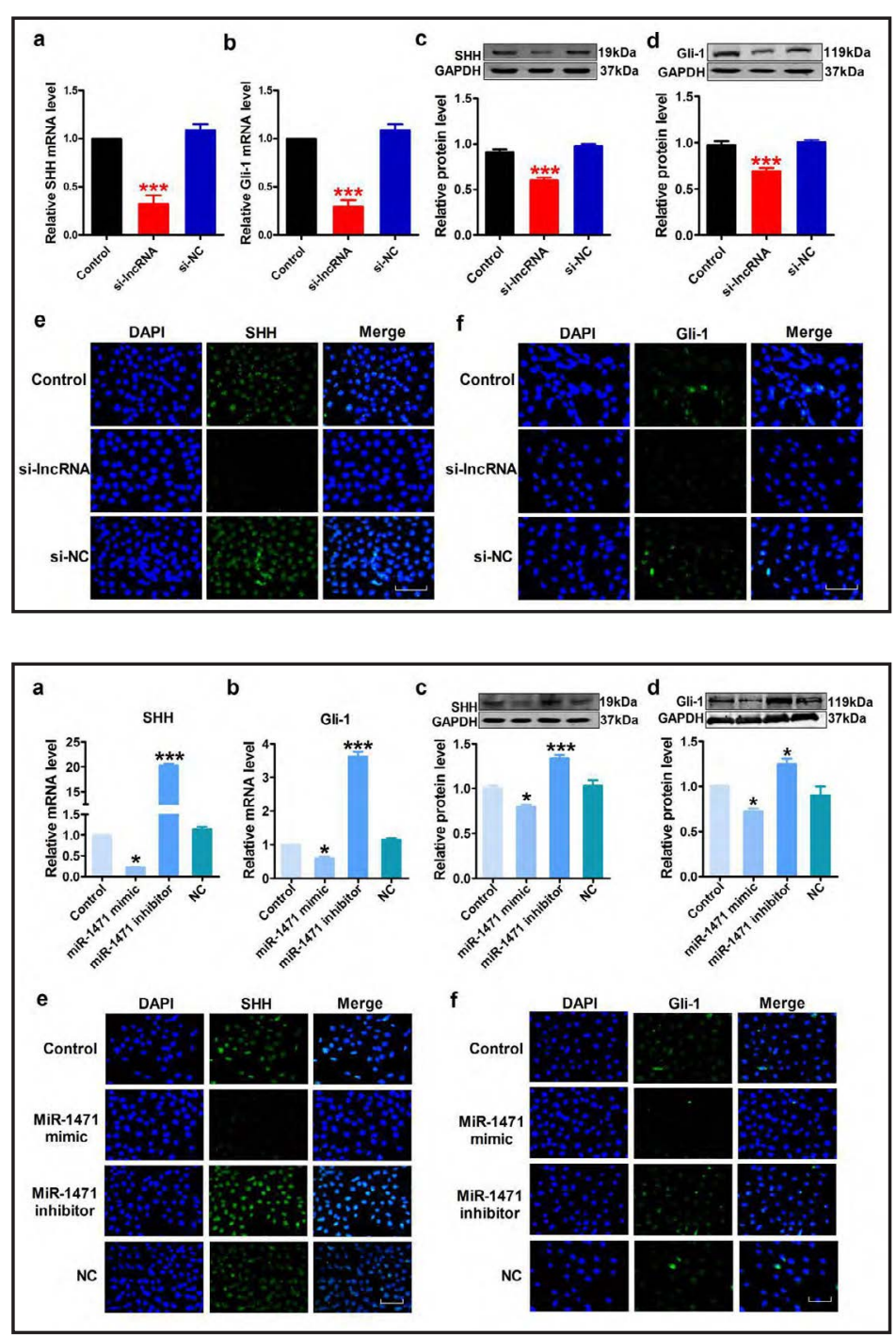
transition (EMT) program is the major result of Gli-1 activation in cancer. We then found the upregulation of epithelial cell marker E-cadherin, and downregulation of mesenchymal cell marker vimentin and $\alpha$-SMA after miR-1471 mimic transfection. While miR-1471 inhibitor transfection caused the opposite results (see online suppl. material, Suppl. Fig. 5). That demonstrated the suppressive effect of miR-1471 on EMT through targeting SHH. Taken together, SHH/Gli-1 signaling pathway is the downstream effector of LOC101930370/miR1471 mediated breast cancer metastasis.

\section{Discussion}

$\mathrm{BC}$ is a clinically challenging disease involving complicated changes in the genome and multiple related signaling pathways [28]. To date, changes of epigenetic modification in cancer related genes have been the major focus [29-31]. Among them, the role of Hedgehog signaling pathway in breast cancer has been recognized long ago. Most of the interest in the Hedgehog signaling pathway in cancer is in its role as a tumor suppressor [18, 32, 33]. SHH ligand is necessary for Hedgehog signaling activation. In BC, SHH has been proved to be overexpressed in tumor tissues and serum of BC patients [34]. Inhibition of SHH contributed to the anti-tumor activity of thiostrepton in $\mathrm{BC}$ [35]. Moreover, its downstream regulator Gli1 , was crucial for invasion of $B C$ and associated with unfavourable overall survival of BC [19, 36]. Consistent with these studies, our research also suggested a remarkable upregulation of SHH and Gli-1 in BC tissues. Downregulation of SHH and Gli-1 by miR-1471 caused the suppression of EMT process in MCF-7 cells. While upregulation of SHH and Gli-1 exerted the opposite effects.

Moreover, it became apparent that ncRNAs were potent regulators of the pathway in this context. The importance of ncRNAs is demonstrated by the fact that a substantial part of genome is devoted to their transcription [24,37]. As the power of miRNA in regulation of mRNA has been explored elaborately, we are beginning to recognize the complex interactions of miRNAs and lncRNAs and ensuing RNA regulatory networks. As the potent upstream regulators of Hedgehog signaling pathway, interaction of miRNAs with lncRNAs revealed in this study discloses the missing links in epigenetic regulation of SHH and provides novel therapeutic strategies for BC.

In our study, we firstly identified the anti-metastatic functions of miR-1471 on MCF-7 cells. Schrauder el al suggested that miR-1471 was downregulated in BC for the first time [8]. However, some studies reported that miR-1471 was overexpressed in lung adenocarcinoma [38] and hepatocellular carcinoma [39]. But the molecular mechanisms of miR-1471 in cancer development were still unknown. Here we also observed a downregulation of miR1471 in BC tissues and proved miR-1471 as a tumor suppressor by targeting SHH dependent Hedgehog signaling pathway. Moreover, gain-of-function as well as loss-of-function experiments also revealed that the proven oncogenesis effects of SHH rely on the miR-1471. Our results showed that miR-1471 significantly downregulated SHH expression in transcript and protein levels. After transfection with miR-1471 mimic, we recorded a trend in SHH downregulation. On the contrary, SHH expression was significantly increased after miR-1471 inhibitor transfection, whereas the expression levels remained unchanged in miR-1471 NCtransfected cells.

Emerging evidences have demonstrated that lncRNAs can function as a natural miRNA sponge, interfere with miRNA pathways and modulate the repression of miRNA targets [40, 41]. LncRNA-H19/MiR-675-5p Pathway mediated the inhibitory effects of Huaier Extract on BC Progression [42]. MALAT1 induced migration and invasion of human BC cells by competitively binding miR-1 with cdc42 [43]. Here we identified LOC101930370 might responsible for the downregulation of miR-1471 in BC. Luciferase assay proved the inhibition effect of LOC101930370 on miR-1471. Real-time PCR showed that LOC101930370 levels were significantly elevated in BC tissues and MCF-7 cells. As expected, LOC101930370 could positively regulate SHH expression through sponging miR-1471 in MCF-7 cells. And 
Liu et al.: LOC101930370/miR-1471 Axis Regulates Breast Cancer Metastasis

knockdown of LOC101930370 by its siRNA inhibited MCF-7 cell metastasis via upregulation of miR-1471. In the meanwhile, the downstream transcriptional factor of SHH, Gli-1, was significantly down-regulated by LOC101930370 siRNA transfection. Nevertheless, further investigation of LOC101930370 may also contribute to understanding of SHH biology and SHH-driven oncogenesis.

In conclusion, miR-1471 exerts anti-metastatic effects by suppression of SHH-dependent Hedgehog signaling pathway in MCF-7 cells. Besides, we identify LOC101930370 as an oncogene, which exerts a crucial role in the proliferation and metastasis in BC. Moreover, for the first time, our study sheds light on the interaction between LOC101930370 and miR-1471, and reveals that LOC101930370 positively regulates the expression of SHH by sponging miR-1471 in BC. Our findings will help to gain knowledge about the molecular mechanisms of Hedgehog signaling pathway in BC and show that RNAi-based approaches may contribute to the development of more effective cancer therapies.

\section{Acknowledgements}

This work was supported by the National Nature Science Foundation of China (81773735, 81570399), Hei Long Jiang Outstanding Youth Science Fund (JJ2017JQ0035) and Hei Long Jiang Postdoctoral Foundation.

\section{Disclosure Statement}

The manuscript is original work that has not been submitted to and is not under consideration for publication by another journal. We confirm that all the listed authors have participated actively in the study and have seen and approved the submitted manuscript. The authors declare to have no competing interests.

\section{References}

1 Yang C, Tabatabaei SN, Ruan X, Hardy P: The Dual Regulatory Role of MiR-181a in Breast Cancer. Cell Physiol Biochem 2017;44:843-856.

2 Jaffee EM, Dang CV, Agus DB, Alexander BM, Anderson KC, Ashworth A, Barker AD, Bastani R, Bhatia S, Bluestone JA, Brawley O, Butte AJ, Coit DG, Davidson NE, Davis M, DePinho RA, Diasio RB, Draetta G, Frazier AL, Futreal A, Gambhir SS, Ganz PA, Garraway L, Gerson S, Gupta S, Heath J, Hoffman RI, Hudis C, HughesHalbert C, Ibrahim R, Jadvar H, Kavanagh B, Kittles R, Le QT, Lippman SM, Mankoff D, Mardis ER, Mayer DK, McMasters K, Meropol NJ, Mitchell B, Naredi P, Ornish D, Pawlik TM, Peppercorn J, Pomper MG, Raghavan D, Ritchie C, Schwarz SW, Sullivan R, Wahl R, Wolchok JD, Wong SL, Yung A: Future cancer research priorities in the USA: a Lancet Oncology Commission. Lancet Oncol 2017;18:e653-e706.

3 Zhang T, Hu Y, Ju J, Hou L, Li Z, Xiao D, Li Y, Yao J, Wang C, Zhang Y, Zhang L: Downregulation of miR-522 suppresses proliferation and metastasis of non-small cell lung cancer cells by directly targeting DENN/ MADD domain containing 2D. Sci Rep 2016;6:19346.

4 O'Bryan S, Dong S, Mathis JM, Alahari SK: The roles of oncogenic miRNAs and their therapeutic importance in breast cancer. Eur J Cancer 2017;72:1-11.

5 Portius D, Sobolewski C, Foti M: MicroRNAs-Dependent Regulation of PPARs in Metabolic Diseases and Cancers. PPAR Res 2017;2017:7058424.

6 Sita-Lumsden A, Dart DA, Waxman J, Bevan CL: Circulating microRNAs as potential new biomarkers for prostate cancer. Br J Cancer 2013;108:1925-1930.

7 Chu C, Liu X, Bai X, Zhao T, Wang M, Xu R, Li M, Hu Y, Li W, Yang L, Qin Y, Yang M, Yan C, Zhang Y: MiR-519d suppresses breast cancer tumorigenesis and metastasis via targeting MMP3. Int J Biol Sci 2018;14:228236. 


\section{Cellular Physiology Cell Physiol Biochem 2018;48:1139-1150 \begin{tabular}{l|l} 
and Biochemistry Published $10.1159 / 000491980$ & $\begin{array}{l}\text { C } 2018 \text { The Author(s). Published by S. Karger AG, Basel } \\
\text { www.karger.com/cpb }\end{array}$
\end{tabular}

Liu et al.: LOC101930370/miR-1471 Axis Regulates Breast Cancer Metastasis

8 Schrauder MG, Strick R, Schulz-Wendtland R, Strissel PL, Kahmann L, Loehberg CR, Lux MP, Jud SM, Hartmann A, Hein A, Bayer CM, Bani MR, Richter S, Adamietz BR, Wenkel E, Rauh C, Beckmann MW, Fasching PA: Circulating micro-RNAs as potential blood-based markers for early stage breast cancer detection. PLoS One 2012; 7:e29770.

-9 Lin EH, Kao YR, Lin CA, Kuo TY, Yang SP, Hsu CF, Chou TY, Ho CC, Wu CW: Hedgehog pathway maintains cell survival under stress conditions, and drives drug resistance in lung adenocarcinoma. Oncotarget 2016;7:24179-24193.

10 Zhao H, Tang H, Xiao Q, He M, Zhao L, Fu Y, Wu H, Yu Z, Jiang Q, Yan Y, Jin F, Wei M: The Hedgehog signaling pathway is associated with poor prognosis in breast cancer patients with the CD44+/CD24 phenotype. Mol Med Rep 2016;14:5261-5270.

11 Diao Y, Azatyan A, Rahman MF, Zhao C, Zhu J, Dahlman-Wright K, Zaphiropoulos PG: Blockade of the Hedgehog pathway downregulates estrogen receptor alpha signaling in breast cancer cells. Oncotarget 2016;7:71580-71593.

12 Habib JG, O'Shaughnessy JA: The hedgehog pathway in triple-negative breast cancer. Cancer Med 2016;5:2989-3006.

13 Flemban A, Qualtrough D: The Potential Role of Hedgehog Signaling in the Luminal/Basal Phenotype of Breast Epithelia and in Breast Cancer Invasion and Metastasis. Cancers (Basel) 2015;7:1863-1884.

14 Hui M, Cazet A, Nair R, Watkins DN, O’Toole SA, Swarbrick A: The Hedgehog signalling pathway in breast development, carcinogenesis and cancer therapy. Breast Cancer Res 2013;15:203.

15 Mishra P, Panda A, Bandyopadhyay A, Kumar H, Mohiddin G: Sonic Hedgehog Signalling Pathway and Ameloblastoma - A Review. J Clin Diagn Res 2015;9:ZE10-13.

16 Du Z, Zhou F, Jia Z, Zheng B, Han S, Cheng J, Zhu G, Huang P: The hedgehog/Gli-1 signaling pathways is involved in the inhibitory effect of resveratrol on human colorectal cancer HCT116 cells. Iran J Basic Med Sci 2016;19:1171-1176.

17 Chun HW, Hong R: Significance of the hedgehog pathway-associated proteins Gli-1 and Gli-2 and the epithelial-mesenchymal transition-associated proteins Twist and E-cadherin in hepatocellular carcinoma. Oncol Lett 2016;12:1753-1762.

18 Di Mauro C, Rosa R, D’Amato V, Ciciola P, Servetto A, Marciano R, Orsini RC, Formisano L, De Falco S, Cicatiello V, Di Bonito M, Cantile M, Collina F, Chambery A, Veneziani BM, De Placido S, Bianco R: Hedgehog signalling pathway orchestrates angiogenesis in triple-negative breast cancers. Br J Cancer 2017;116:14251435.

19 Song Y, Zhang J, Tian T, Fu X, Wang W, Li S, Shi T, Suo A, Ruan Z, Guo H, Yao Y: SET7/9 inhibits oncogenic activities through regulation of Gli-1 expression in breast cancer. Tumour Biol 2016;37:9311-9322.

20 Yao J, Xu T, Tian T, Fu X, Wang W, Li S, Shi T, Suo A, Ruan Z, Guo H, Yao Y: Tripartite motif 16 suppresses breast cancer stem cell properties through regulation of Gli-1 degradation via the ubiquitin-proteasome pathway. Oncol Rep 2016;35:1204-1212.

21 Lu L, Wu M, Zhao F, Fu W, Li W, Li X, Liu T: Prognostic and clinicopathological value of Gli-1 expression in gastric cancer: A meta-analysis. Oncotarget 2016;7:69087-69096.

$22 \mathrm{Hu} \mathrm{H}$, Shu M, He L, Yu X, Liu X, Lu Y, Chen Y, Miao X, Chen X: Epigenomic landscape of 5-hydroxymethylcytosine reveals its transcriptional regulation of lncRNAs in colorectal cancer. Br J Cancer 2017;116:658-668.

23 Pan Y, Li C, Chen J, Zhang K, Chu X, Wang R, Chen L: The Emerging Roles of Long Noncoding RNA ROR (lincRNA-ROR) and its Possible Mechanisms in Human Cancers. Cell Physiol Biochem 2016;40:219-229.

-24 Zhao L, Sun H, Kong H, Chen Z, Chen B, Zhou M: The Lncrna-TUG1/EZH2 Axis Promotes Pancreatic Cancer Cell Proliferation, Migration and EMT Phenotype Formation Through Sponging Mir-382. Cell Physiol Biochem 2017;42:2145-2158.

25 Cai B, Ma W, Bi C, Yang F, Zhang L, Han Z, Huang Q, Ding F, Li Y, Yan G, Pan Z, Yang B, Lu Y: Long noncoding RNA H19 mediates melatonin inhibition of premature senescence of c-kit(+) cardiac progenitor cells by promoting miR-675. J Pineal Res 2016;61:82-95.

26 Gee HE, Buffa FM, Camps C, Ramachandran A, Leek R, Taylor M, Patil M, Sheldon H, Betts G, Homer J, West C, Ragoussis J, Harris AL: The small-nucleolar RNAs commonly used for microRNA normalisation correlate with tumour pathology and prognosis. Br J Cancer 2011;104:1168-1177. 


\section{Cellular Physiology Cell Physiol Biochem 2018;48:1139-1150 \begin{tabular}{l|l} 
and Biochemistry Published online: July 24, 2018 & $\begin{array}{l}\text { C) } 2018 \text { The Author(s). Published by S. Karger AG, Basel } \\
\text { www.karger.com/cpb }\end{array}$ \\
\hline
\end{tabular}}

Liu et al.: LOC101930370/miR-1471 Axis Regulates Breast Cancer Metastasis

27 Sagai T, Amano T, Maeno A, Kimura T, Nakamoto M, Takehana Y, Naruse K, Okada N, Kiyonari H, Shiroishi T: Evolution of Shh endoderm enhancers during morphological transition from ventral lungs to dorsal gas bladder. Nat Commun 2017;8:14300.

28 Nielsen FC, van Overeem Hansen T, Sorensen CS: Hereditary breast and ovarian cancer: new genes in confined pathways. Nat Rev Cancer 2016;16:599-612.

29 Huarte M: The emerging role of IncRNAs in cancer. Nat Med 2015;21:1253-1261.

-30 Pfister SX, Ashworth A: Marked for death: targeting epigenetic changes in cancer. Nat Rev Drug Discov 2017;16:241-263.

-31 Feinberg AP, Koldobskiy MA, Gondor A: Epigenetic modulators, modifiers and mediators in cancer aetiology and progression. Nat Rev Genet 2016;17:284-299.

32 Takebe N, Miele L, Harris PJ, Jeong W, Bando H, Kahn M, Yang SX, Ivy SP: Targeting Notch, Hedgehog, and Wnt pathways in cancer stem cells: clinical update. Nat Rev Clin Oncol 2015;12:445-464.

-33 Noman AS, Uddin M, Chowdhury AA, Nayeem MJ, Raihan Z, Rashid MI, Azad AK, Rahman ML, Barua D, Sultana A, Shirin A, Ferdous J, Parag RR, Rahman SM, Mahmud MR, Jerin CS, Jahan N, Siddiqua A, Ara T, Sabur EB, Alam SS, Baidya S, Akther S, Rahman MZ, Banu T, Murugan AK, Sabri S, Islam SMS, Karakas B, Aboussekhra A, Yeger H, Farhat WA, Islam SS: Serum sonic hedgehog (SHH) and interleukin-(IL-6) as dual prognostic biomarkers in progressive metastatic breast cancer. Sci Rep 2017;7:1796.

-34 Riaz SK, Khan JS, Shah STA, Wang F, Ye L, Jiang WG, Malik MFA: Involvement of hedgehog pathway in early onset, aggressive molecular subtypes and metastatic potential of breast cancer. Cell Commun Signal 2018;16:3.

-35 Yang N, Zhou TC, Lei XX, Wang C, Yan M, Wang ZF, Liu W, Wang J, Ming KH, Wang BC, Xu BL, Liu Q: Inhibition of Sonic Hedgehog Signaling Pathway by Thiazole Antibiotic Thiostrepton Attenuates the CD44+/CD24Stem-Like Population and Sphere-Forming Capacity in Triple-Negative Breast Cancer. Cell Physiol Biochem 2016;38:1157-1170.

-36 ten Haaf A, Bektas N, von Serenyi S, Losen I, Arweiler EC, Hartmann A, Knuchel R, Dahl E: Expression of the glioma-associated oncogene homolog (GLI) 1 in human breast cancer is associated with unfavourable overall survival. BMC Cancer 2009;9:298.

-37 Bresson S, Tollervey D: Surveillance-ready transcription: nuclear RNA decay as a default fate. Open Biol 2018;8.

-38 Zhao C, Xu Y, Zhang Y, Tan W, Xue J, Yang Z, Zhang Y, Lu Y, Hu X: Downregulation of miR-145 contributes to lung adenocarcinoma cell growth to form brain metastases. Oncol Rep 2013;30:2027-2034.

39 Zha R, Guo W, Zhang Z, Qiu Z, Wang Q, Ding J, Huang S, Chen T, Gu J, Yao M, He X: Genome-wide screening identified that miR-134 acts as a metastasis suppressor by targeting integrin beta1 in hepatocellular carcinoma. PLoS One 2014;9:e87665.

40 Wu D, Li Y, Zhang H, Hu X: Knockdown of Lncrna PVT1 Enhances Radiosensitivity in Non-Small Cell Lung Cancer by Sponging Mir-195 Cell Physiol Biochem 2017;42:2453-2466.

-41 Zhang Y, Liu X, Bai X, Lin Y, Li Z, Fu J, Li M, Zhao T, Yang H, Xu R, Li J, Ju J, Cai B, Xu C, Yang B: Melatonin prevents endothelial cell pyroptosis via regulation of long noncoding RNA MEG3/miR-223/NLRP3 axis. J Pineal Res 2018;64.

-42 Wang J, Wang X, Chen T, Jiang L, Yang Q: Huaier Extract Inhibits Breast Cancer Progression Through a LncRNA-H19/MiR-675-5p Pathway. Cell Physiol Biochem 2017;44:581-593.

43 Chou J, Wang B, Zheng T, Li X, Zheng L, Hu J, Zhang Y, Xing Y, Xi T: MALAT1 induced migration and invasion of human breast cancer cells by competitively binding miR-1 with cdc42. Biochem Biophys Res Commun 2016;472:262-269. 